\title{
Correction to: investigating regional distribution for maximum daily rainfall in arid regions: case study in Saudi Arabia
}

\author{
Wael M. Abdeen ${ }^{1} \cdot$ Ayman G. Awadallah ${ }^{2}$ - Nagy A. Hassan ${ }^{3}$
}

Published online: 10 July 2020

(C) Saudi Society for Geosciences 2020

Correction to: Arabian Journal of Geosciences (2020) 13:501. https://doi.org/10.1007/s12517-020-05413-8

The original version of this paper was published with error. Tables 1, 2 and 3 were incorrectly process and the corresponding author should be "Ayman G. Awadallah".

The original article has been corrected.

The online version of the original article can be found at https://doi.org/ 10.1007/s12517-020-05413-8

Ayman G. Awadallah

aawadallah@darcairo.com; ayman.awadallah@dar.com

Wael M. Abdeen

waelmagdyabdeen80@gmail.com

Nagy A. Hassan

ngyhassan@yahoo.com
1 Irrigation and Hydraulics Department, Faculty of Engineering, Ain Shams University, Cairo, Egypt

2 Civil Engineering Department, Faculty of Engineering, Fayoum University, Fayoum, Egypt

3 Irrigation and Hydraulics Department Faculty of Engineering, Ain Shams University, Cairo, Egypt 
Table 1 Ranking of the eight distributions based on $A I C$ and $B I C$ for individual station

\begin{tabular}{|c|c|c|c|c|c|c|c|c|c|}
\hline Region & No. of rainfall stations & LN3 & Gamma & Generalized gamma & GEV & Gumbel & Log-Pearson III & Pareto & Pearson III \\
\hline Al Jawf & 10 & 6 & 1 & 5 & 7 & 3 & 2 & 8 & 4 \\
\hline Aseer & 25 & 6 & 1 & 5 & 4 & 2 & 3 & 8 & 7 \\
\hline Al Baha & 8 & 6 & 1 & 4 & 7 & 2 & 3 & 8 & 5 \\
\hline Eastern Province & 20 & 6 & 1 & 4 & 7 & 3 & 2 & 8 & 5 \\
\hline Jazan & 29 & 6 & 1 & 5 & 3 & 2 & 4 & 8 & 7 \\
\hline Hail & 17 & 7 & 1 & 4 & 5 & 3 & 2 & 8 & 6 \\
\hline Madinah & 32 & 7 & 1 & 5 & 6 & 2 & 3 & 4 & 8 \\
\hline Makkah & 49 & 6 & 1 & 4 & 5 & 2 & 3 & 8 & 7 \\
\hline Northern Border & 2 & 3 & 2 & 5 & 4 & 6 & 1 & 7 & 8 \\
\hline Najran & 4 & 4 & 1 & 3 & 6 & 5 & 2 & 8 & 7 \\
\hline Qaseem & 20 & 8 & 1 & 6 & 5 & 2 & 3 & 4 & 7 \\
\hline Riyadh & 43 & 7 & 1 & 4 & 6 & 3 & 2 & 8 & 5 \\
\hline Tabouk & 10 & 5 & 1 & 3 & 7 & 6 & 2 & 4 & 8 \\
\hline Sum of rankings & & 77 & 14 & 57 & 72 & 41 & 32 & 91 & 84 \\
\hline
\end{tabular}

Table 2 Number of rainfall stations within each homogeneous group with their corresponding $p$ values

\begin{tabular}{lll}
\hline Homogeneous group & No. of rainfall stations & P value \\
\hline Group1 (Hail, Qaseem, Al-Jawf, Northern Border, & 89 & 0.146 \\
$\quad$ Eastern Province, Riyadh) & & 0.120 \\
Group 2 (Makkah, Madinah) & 49 & 0.054 \\
Group 3 (Najran, Aseer, Al Baha, Jazan) & 51 & 0.073 \\
Makkah1 & 22 & 0.125 \\
Tabouk & 14 & 0.112 \\
Riyadh1 & 22 & 0.085 \\
Jazan1 & 20 & \\
Total & $267^{\mathrm{a}}$ & \\
\hline
\end{tabular}

${ }^{\text {a }}$ With, as previously mentioned, two stations removed from the total of 269 available rainfall stations

Table 3 Growth curves values and ratios of the various homogeneous groups

\begin{tabular}{|c|c|c|c|c|c|c|c|c|c|c|c|c|c|}
\hline \multirow[t]{2}{*}{$\begin{array}{l}\text { Return period } \\
\text { (years) }\end{array}$} & \multicolumn{7}{|c|}{ Group name } & \multicolumn{3}{|c|}{$\begin{array}{l}\text { Ratio of X return period/2- } \\
\text { year return period (all groups) }\end{array}$} & \multicolumn{3}{|c|}{$\begin{array}{l}\text { Ratio of } X \text { return period/2-year } \\
\text { return period }(\mathrm{Gr} 1, \mathrm{Gr} 2, \mathrm{Gr} 3 \text {, } \\
\text { Mak1) }\end{array}$} \\
\hline & Group 1 & Group 2 & Group 3 & Gizan & Makkah 1 & Riyadh 1 & Tabouk & $\mathrm{Av}$ & Std Dev & $\mathrm{CoV}$ & $\mathrm{Av}$ & Std Dev & $\mathrm{CoV}$ \\
\hline 10,000 & 6.36 & 5.92 & 6.70 & 3.13 & 5.7 & 3.96 & 11.1 & 7.385 & 0.784 & 0.106 & 7.583 & 0.748 & 0.099 \\
\hline 2000 & 5.34 & 5.20 & 5.39 & 2.90 & 4.8 & 3.55 & 8.63 & 6.189 & 0.650 & 0.105 & 6.361 & 0.623 & 0.098 \\
\hline 1000 & 4.90 & 4.85 & 4.85 & 2.78 & 4.42 & 3.36 & 7.63 & 5.673 & 0.593 & 0.105 & 5.832 & 0.570 & 0.098 \\
\hline 500 & 4.46 & 4.49 & 4.34 & 2.66 & 4.03 & 3.16 & 6.67 & 5.163 & 0.538 & 0.104 & 5.307 & 0.518 & 0.098 \\
\hline 200 & 3.87 & 3.98 & 3.70 & 2.47 & 3.52 & 2.88 & 5.47 & 4.488 & 0.464 & 0.103 & 4.612 & 0.448 & 0.097 \\
\hline 100 & 3.42 & 3.57 & 3.23 & 2.32 & 3.14 & 2.65 & 4.61 & 3.976 & 0.408 & 0.103 & 4.081 & 0.395 & 0.097 \\
\hline 50 & 2.97 & 3.14 & 2.78 & 2.14 & 2.75 & 2.4 & 3.8 & 3.462 & 0.353 & 0.102 & 3.551 & 0.343 & 0.097 \\
\hline 25 & 2.52 & 2.68 & 2.35 & 1.95 & 2.36 & 2.13 & 3.05 & 2.949 & 0.298 & 0.101 & 3.018 & 0.290 & 0.096 \\
\hline 20 & 2.37 & 2.52 & 2.21 & 1.88 & 2.24 & 2.04 & 2.81 & 2.779 & 0.280 & 0.101 & 2.840 & 0.272 & 0.096 \\
\hline 10 & 1.92 & 2.03 & 1.80 & 1.65 & 1.85 & 1.74 & 2.12 & 2.262 & 0.225 & 0.099 & 2.305 & 0.219 & 0.095 \\
\hline 5 & 1.46 & 1.52 & 1.39 & 1.39 & 1.45 & 1.42 & 1.49 & 1.736 & 0.169 & 0.097 & 1.757 & 0.165 & 0.094 \\
\hline 3 & 1.12 & 1.13 & 1.10 & 1.16 & 1.14 & 1.15 & 1.05 & 1.341 & 0.129 & 0.096 & 1.351 & 0.126 & 0.093 \\
\hline 2 & 0.826 & 0.796 & 0.842 & 0.935 & 0.88 & 0.901 & 0.718 & 1.000 & & & 1.000 & & \\
\hline
\end{tabular}

*Av weighted average, Std Dev weighted standard deviation, $C o V$ weighted coefficient of variation 